\title{
ON A CLASS OF OPERATORS
}

GEORGE H. ORLAND ${ }^{1}$

1. Introduction. There is a large class of bounded operators on Hilbert space whose resolvents have first order rate-of-growth. By such an operator is meant one whose resolvent at a point $\lambda$ has its norm dominated by the reciprocal of the distance from $\lambda$ to the convex hull of the spectrum of the operator. They can be given a geometrical characterization, namely that their numerical ranges coincide with the convex hulls of their spectra. It will also be shown that an operator is in this class when "enough" points of its numerical range have their moduli equal to the norm of the operator. Every normal operator is in this class. In addition, many nonnormal operators are in this class. Using results of Halmos and Bram $[4 ; 1]$, it can be shown that every subnormal operator is also in it.

The author wishes to express his gratitude to Professor Frantisek Wolf for his encouragement and helpful suggestions during the preparation of this paper.

2. Some preliminaries. The notation and terminology to be used are for the most part standard and will tend to conform with [3] and [8]. However some items should be mentioned explicitly. Only bounded operators on complex Hilbert spaces are considered; $\sigma(T)$, $\Sigma(T),|\sigma(T)|, \rho(T)$, and $R_{\lambda}(T)$ denote respectively the spectrum of the operator $T$, the (automatically closed) convex hull of $\sigma(T)$, the spectral radius of $T$, the resolvent set of $T$, and $(T-\lambda)^{-1}$ for $\lambda \in \rho(T)$. When there is no possibility of confusion, these symbols will be simplified to $\sigma, \Sigma$, etc. The distance between a point $\lambda$ and a set $S$ (in the complex plane) is indicated as $d(\lambda, S)$.

Let $T$ be an operator on a Hilbert space $H$. Let $W(T)$ be the closure of the set of complex numbers, $\{(T x, x): x \in H$ and $\|x\|=1\}$; it is the numerical range of $T$. The numerical radius of $T$, denoted by $|W(T)|$, is the $\sup \{|\lambda|: \lambda \in W(T)\}$. Certain facts are known about $W(T)$, e.g. see [8]. Among them are: $W(T)$ is a convex subset of the complex plane; $\sigma(T) \subset W(T)$; if $T$ is normal then $W(T)=\Sigma(T) ; \frac{1}{2}\|T\| \leqq|W(T)|$ $\leqq\|T\|$; and $W(\alpha T+\beta)=\alpha W(T)+\beta$, where $\alpha$ and $\beta$ are complex numbers.

For any operator $T$ it is always true $\left[2\right.$, p. 566] that $[d(\lambda, \sigma)]^{-1}$

Received by the editors March 2, 1962 and, in revised form, October 5, 1962.

1 This work was supported by the National Science Foundation grant 10098. It is part of the author's doctoral dissertation prepared under the direction of Professor Frantisek Wolf at the University of California, Berkeley. 
$\leqq\left\|R_{\lambda}\right\|$. In the case of a normal operator the opposite inequality also holds (by a simple application of the spectral theorem). The operators considered in this paper will satisfy a slightly weakened inequality, namely that $\left\|R_{\lambda}\right\| \leqq[d(\lambda, \Sigma)]^{-1}$ for all $\lambda \in \Sigma$. Also for any operator $T$ and $\lambda \notin \Sigma$ the fact that $\left\|R_{\lambda}\right\| \leqq[d(\lambda, \Sigma)]^{-1}$ if and only if $d(\lambda, \Sigma) \cdot\|y\| \leqq\|T y-\lambda y\|$ for all $y \in H$, will be used frequently.

LEMma 1. Let $T$ be an operator satisfying $\left\|R_{\lambda}(T)\right\| \leqq[d(\lambda, \Sigma(T))]^{-1}$ for some $\lambda \notin \Sigma(T)$. Let $\alpha \neq 0$ and $\beta$ be complex numbers. Then $z \rightarrow \alpha z+\beta$ maps the complement of $\Sigma(T)$ onto the complement of $\Sigma(\alpha T+\beta)$, and $\alpha T+\beta$ satisfies

$$
\left\|R_{\alpha \lambda+\beta}(\alpha T+\beta)\right\| \leqq \frac{1}{d(\alpha \lambda+\beta, \Sigma(\alpha T+\beta))} .
$$

PRoof. The complex plane is mapped onto itself by $z \rightarrow \alpha z+\beta$, since $\alpha \neq 0$. As is well known, $\sigma(T)$ is mapped onto $\sigma(\alpha T+\beta)$ and consequently $\Sigma(T)$ is mapped onto $\Sigma(\alpha T+\beta)$. The first conclusion then follows by taking complements.

From the hypothesis we get $d(\lambda, \Sigma(T)) \cdot\|y\| \leqq\|T y-\lambda y\|$ for all $y$ so that

$$
\begin{aligned}
|\alpha| \cdot d(\lambda, \Sigma(T)) \cdot\|y\| & =d(\alpha \lambda, \alpha \Sigma(T)) \cdot\|y\|=d(\alpha \lambda, \Sigma(\alpha T)) \cdot\|y\| \\
& =d(\alpha \lambda+\beta, \Sigma(\alpha T)+\beta) \cdot\|y\| \\
& =d(\alpha \lambda+\beta, \Sigma(\alpha T+\beta)) \cdot\|y\| \leqq|\alpha| \cdot\|T y-\lambda y\| \\
& =\|(\alpha T+\beta) y-(\alpha+\beta) y\| .
\end{aligned}
$$

This gives the second conclusion.

Lemma 2. $\left\|R_{\lambda}\right\| \leqq 1 / \lambda$ for all $\lambda>0$ if and only if $\operatorname{Re} W(T) \leqq 0$.

Proof. For all $y$ and $\lambda>0, \lambda\|y\| \leqq\|(T-\lambda) y\|$ if and only if for all $y$ and $\lambda>0, \lambda^{2}\|y\|^{2} \leqq\|T y\|^{2}+\lambda^{2}\|y\|^{2}-2 \lambda \operatorname{Re}(T y, y)$ if and only if for all $y$ and $\lambda>0,2 \lambda \operatorname{Re}(T y, y) \leqq\|T y\|^{2}$ if and only if for all $y, \operatorname{Re}(T y, y)$ $\leqq 0$ if and only if $\operatorname{Re} W(T) \leqq 0$.

3. Main results. Toivo Nieminen kindly communicated the following theorem to me. It is published here with his permission [7].

THEOREM 1. If $\sigma(T)$ is real and $\left\|R_{\lambda}\right\| \leqq|\lambda|^{-1}$ for all nonzero, purely imaginary $\lambda$, then $T$ is self-adjoint.

Proof. Let $\lambda=i \mu$ so $\left\|R_{i \mu}\right\| \leqq|\mu|^{-1}$. Therefore, for all $y \in H$ and $\mu$ nonzero real, $|\mu| \cdot\|y\| \leqq\|T y-i \mu y\|$ so that $\mu^{2}\|y\|^{2} \leqq\|T y\|^{2}+\mu^{2}\|y\|^{2}$ $-2 \mu \operatorname{Im}(T y, y)$ or $2 \mu \operatorname{Im}(T y, y) \leqq\|T y\|^{2}$. Hence $\operatorname{Im}(T y, y)=0$ and $(T y, y)$ is real. This is equivalent to the self-adjointness of $T$. 
THEOREM 2. If $T$ is an operator satisfying $\left\|R_{\lambda}\right\| \leqq[d(\lambda, \Sigma)]^{-1}$ for all $\lambda \notin \Sigma(T)$, then $W(T)=\Sigma(T)$.

Proof. Since $\Sigma(T) \subset W(T)$, only the opposite inclusion must be shown. Let $L$ be a line-of-support for $\Sigma(T)$. The closed half-plane so determined which contains $\Sigma(T)$ will be shown to contain $W(T)$. Now $L$ touches $\Sigma(T)$ at a point $\gamma$. There are complex numbers $\alpha$ and $\beta$ with $|\alpha|=1$, such that the rigid motion $z \rightarrow \alpha z+\beta$ takes $\gamma$ into the origin, $L$ into the $y$-axis, and $\Sigma(T)$ into part of the left closed halfplane. Under this mapping $\Sigma(T) \rightarrow \alpha \Sigma(T)+\beta=\Sigma(\alpha T+\beta)$ and $W(T)$ $\rightarrow \alpha W(T)+\beta=W(\alpha T+\beta)$. Application of Lemma 1 yields

$$
\left\|R_{\alpha \lambda+\beta}(\alpha T+\beta)\right\| \leqq \frac{1}{d(\alpha \lambda+\beta, \Sigma(\alpha T+\beta))}
$$

for all $\alpha \lambda+\beta \notin \Sigma(\alpha T+\beta)$. Now $\alpha \lambda+\beta$ may be taken strictly positive so that the hypothesis of Lemma 2 is satisfied. Therefore $\operatorname{Re} W(\alpha T+\beta)$ $\leqq 0$. By the rigidity of the mapping, $W(T)$ must initially have been on the same side of $L$ as $\Sigma(T)$.

Somewhat surprising is the fact that the converse of Theorem 2 is also true.

TheOREM 3. If $T$ is an operator with $W(T)=\Sigma(T)$ then $\left\|R_{\lambda}\right\|$ $\leqq[d(\lambda, \Sigma)]^{-1}$ for all $\lambda \notin \Sigma(T)$.

Proof. Take any $\lambda \notin W(T)=\Sigma(T)$. To each such $\lambda$ there corresponds a unique $\gamma \in W(T)$ such that $d(\lambda, W(T))=|\lambda-\gamma|$. The line $L$ through $\gamma$ and perpendicular to the line joining $\lambda$ and $\gamma$ is a lineof-support for $W(T)$. There are complex numbers $\alpha$ and $\beta$ with $|\alpha|=1$ such that the rigid motion, $z \rightarrow \alpha z+\beta$, carries $\gamma$ onto the origin, $L$ onto the $y$-axis, $\lambda$ onto $|\lambda-\gamma|$, and $W(T)$ into the closed left halfplane. This maps $W(T)=\Sigma(T)$ onto $W(\alpha T+\beta)=\Sigma(\alpha T+\beta)$. By Lemma 2

$$
\left\|R_{|\lambda-\gamma|}(\alpha T+\beta)\right\| \leqq \frac{1}{|\lambda-\gamma|}=\frac{1}{d(|\lambda-\gamma|, \Sigma(\alpha T+\beta))} .
$$

Now $z \rightarrow \alpha z+\beta$ has an inverse mapping which is of the same form. Use Lemma 1 on this last inequality and the inverse map. This yields $\left\|R_{\lambda}(T)\right\| \leqq[d(\lambda, \Sigma(T))]^{-1}$.

There is also the following relationship.

ThEOREM 4. $|\sigma(T)|=\|T\|$ if and only if $|W(T)|=\|T\|$.

PROOF. The forward implication is clear from the fact that $|\sigma(T)|$ $\leqq|W(T)| \leqq\|T\|$. 
Now let $\lambda \in W(T)$ and $|\lambda|=\|T\|$. If $\lambda$ is to be in $\sigma(T)$ then it must be on the boundary of $\sigma(T)$ and so be in the approximate point spectrum. Hence a sequence $\left\{x_{n}\right\}$ of unit vectors must be produced such that $\left\|(T-\lambda) x_{n}\right\|^{2} \rightarrow 0$. But $\left\|(T-\lambda) x_{n}\right\|^{2}=\left\|T x_{n}\right\|^{2}+|\lambda|^{2}-$ $2 \operatorname{Re} \bar{\lambda}\left(T x_{n}, x_{n}\right) \leqq 2|\lambda|^{2}-2 \operatorname{Re} \bar{\lambda}\left(T x_{n}, x_{n}\right)$. Now choose $\left\{x_{n}\right\}$ such that $\left(T x_{n}, x_{n}\right) \rightarrow \lambda$. This does the trick and $\lambda \in \sigma(T)$.

Corollary. If $\lambda \in W(T)$ and $|\lambda|=\|T\|$ then $\lambda \in \sigma(T)$.

Before proving the next theorem, some preliminary results regarding plane convex sets must be discussed. First a notion related to that of extreme point and exposed extreme point must be defined. Let $C$ be a compact convex subset of the plane. A point $p \in C$ is bare if there is a circle through $p$ such that no points of $C$ lie outside this circle.

Lemma 3. Let $C$ be a nonempty compact convex subset of the plane, and let $S$ be the collection of all of its bare points. Then $C$ is the closed convex hull of $S$.

Proof. (This proof is a modification of one suggested by Lester $\mathrm{E}$. Dubins.) If $C$ consists of one point, this point is in $S$. If $C$ consists of more than one point its diameter is assumed at two distinct points. That these points are in $S$ can be seen by considering a circle centered at one and passing through the other. Thus $S$ is nonempty. Let $D$ be the closed convex hull of $S$, so $D \subset C$. Suppose $D \neq C$. Then there is a closed half-plane $P$ which contains $D$ and whose boundary, $L$, is a line-of-support for $D$, while the complementary open half-plane contains a point $p \in C-D$. Let $M$ be a line through $p$ and parallel to $L$. There is a circle containing $P \cap C$ in its interior and disjoint from $M$. Let its center be $q$. Of all circles centered at $q$ and containing $C$ there is a smallest which touches $C$ at a point $r$. Then $r$ is a bare point which necessarily is not in $D$, a contradiction.

The next theorem states a sufficient "geometrical" condition for an operator to have a resolvent with first order rate-of-growth.

THEOREM 5. Let $|\lambda|=\|T\|$ for each $\lambda \in W(T)$ which is a bare point. Then $W(T)=\Sigma(T)$.

Proof. It is only necessary to show that $\Sigma(T) \supset W(T)$, since the opposite inclusion is always automatically true. Via an invocation of Lemma 3, it is sufficient to show that each bare point of $W(T)$ is in $\sigma(T)$. This follows from the hypothesis and the corollary to Theorem 4.

(Added October 5, 1962. The referee has kindly pointed out that 
(i) Theorem 4 is included in Proposition 7, p. 34, and Theorem 4, p. 32 of [5], and that the "only if" part is well known, and (ii) the "if" part of Lemma 2 is included in Lemma 3.1, p. 686 of [6].

\section{REFERENCES}

1. J. Bram, Subnormal operators, Duke Math. J. 22 (1955), 75-94.

2. N. Dunford and J. T. Schwartz, Linear operators. I, Interscience, New York, 1958.

3. P. R. Halmos, Introduction to Hilbert space and the theory of spectral multiplicity, Chelsea Publishing Company, New York, 1951.

4. - Spectra and spectral manifolds, Ann. Soc. Polon. Math. 25 (1952), 43-49. 24-43.

5. G. Lumer, Semi-inner-product spaces, Trans. Amer. Math. Soc. 100 (1961),

6. G. Lumer and R. S. Phillips, Dissipative operators on a Banach space, Pacific J. Math. 11 (1961), 679-698.

7. T. Nieminen, $A$ condition for the selfadjointness of a linear operator, Ann. Acad. Sci. Fenn. Ser. A I No. 316 (1962), 5 pp.

8. M. H. Stone, Linear transformations in Hilbert space and their applications to analysis, Amer. Math. Soc. Colloq. Publ. Vol. 15, Amer. Math. Soc., Providence, R. I., 1932.

University of California, Berkeley 